\title{
Electroluminescence and electrical transport in poly(3-octylthiophene) diodes
}

\author{
D. Braun, G. Gustafsson, D. McBranch, and A. J. Heeger \\ Institute for Polymers and Organic Solids, University of California at Santa Barbara, Santa Barbara, \\ California 93106
}

\begin{abstract}
We report visible light emission from diodes made from poly(3-octylthiophene). Use of a soluble derivative of polythiophene allows fabrication of the light emitting diodes by casting the polymer film from solution with no subsequent processing or heat treatment required. The devices emit dim red-orange light with relatively low external quantum efficiencies, below $2.5 \times 10^{-5}$ photons per electron at room temperature. Electrical characterization reveals diode behavior with rectification ratios greater than $10^{2}$. The temperature dependence indicates that tunneling phenomena dominate the charge injection.
\end{abstract}

\section{INTRODUCTION}

The results of Burroughes et al. ${ }^{1}$ first demonstrated electroluminescence from diodes fabricated from poly (paraphenylene vinylene) (PPV). Subsequent experiments used soluble derivatives of PPV; ${ }^{2}$ the diodes exhibit good brightness with quantum efficiencies as high as $1 \%$ (photons per electron). There are more recent reports of electroluminescence in PPV copolymers and derivatives, ${ }^{3-5}$ polyaniline, ${ }^{5}$ poly(3-alkylthiophenes) ${ }^{6,7}$ and poly(paraphenylene). ${ }^{8}$

This work describes the fabrication of light-emitting diode structures using poly (3-octylthiophene) (P3OT) as the semiconducting polymer and presents the initial characterization of their electrical and electroluminescent properties.

\section{EXPERIMENTAL}

The light emitting diodes (LEDs) consist of an electron-injecting metal contact on the front surface of a P3OT film prepared by spin-casting onto an indium/tinoxide (ITO) coated glass substrate. ITO forms the holeinjecting contact. Use of a polymer which is soluble in the semiconducting state makes the processing sequence quite simple. Spin-casting from tetrahydrofuran solutions containing 3\% P3OT by weight results in P3OT film thicknesses (measured by a Dektak stylus profilometer) of $4000-6000 \AA( \pm 800 \AA)$. The wide range in film thickness results both from sample-to-sample variation and from ripples a few hundred angstroms high that often arise when spinning films from low boiling point solvents. The rectifying calcium or indium contacts are deposited on top of the polymer films by vacuum evaporation at pressures below $2 \times 10^{-6}$ Torr, yielding active areas of $0.1 \mathrm{~cm}^{2}$. Indium solder or silver paint provide contact between electrodes and external wires. Electrical measurements are performed by a Keithley 236 Source-Measure Unit, and a calibrated silicon photodiode simultaneously collects the emitted light. Spectroscopic measurements use a single-grating monochromator followed by a photodiode array as detector. Spectra are corrected for the spectral response of the monochromator and collecting optics by normalization to the response of a calibrated tungsten lamp. For temperature dependence measurements, a copper block containing a sapphire window provides thermal contact from the sample to a gas flow cryogenic system with temperature control maintained by computer. All processing steps and device characterization are carried out in nitrogen or vacuum atmosphere.

\section{DEVICE CHARACTERISTICS}

Figure 1 shows the room temperature electroluminescence (EL) spectrum obtained with $45 \mathrm{~V}$ forward bias on a calcium/P3OT/ITO LED. Positive bias is defined as positive voltage applied to the ITO contact. The EL peaks below the energy gap $\left(E_{\mathrm{g}} \approx 2.1 \mathrm{eV}\right)$, as expected from the excited state of the polaron-exciton (also called a neutral bipolaron) observed in polymers with nondegenerate ground states such as PPV and its soluble derivatives. ${ }^{9}$ The vibronic structure observed in low temperature photoluminescence (PL) spectra ${ }^{10}$ is not resolved in the room temperature EL spectrum of Fig. 1, but the EL spectrum is similar to the broad room temperature PL spectra obtained from other poly (3-alkylthiophenes). ${ }^{11,12}$ The vibronic features arise from phonon emission, and room temperature luminescence generally smears out the vibronic features compared to low temperatures. For LEDs made from $\mathrm{PPV}^{1}$ and a soluble derivatives of PPV, ${ }^{2}$ the EL spectra and the PL spectra are also quite similar.

Figure 2 plots the current flow through the device and the luminance as a function of bias voltage for the calcium/ P3OT/ITO diode. While ramping the applied bias, orangered light becomes visible to the eye above $16 \mathrm{~V}$ forward bias; no light is observed under reversed bias. Above $10 \mathrm{~V}$, the ratio of forward to reverse current ranges from 7500 to 300.

Figure 3 displays the luminance as a function of current flow under increasing forward bias. The slightly superlinear characteristic indicates that the radiative efficiency increases with current, suggesting quenching of nonradiative recombination possibly due to trap-filling or saturating of leakage current pathways. The quantum effi- 


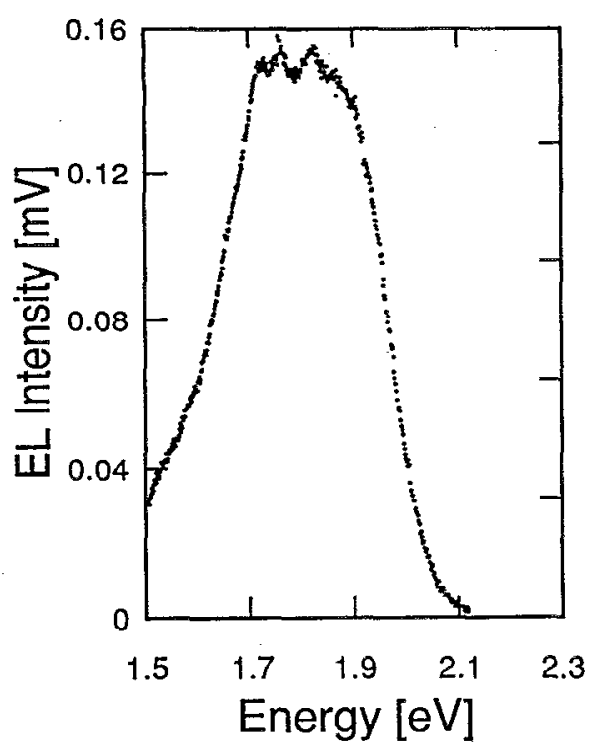

FIG. 1. Electroluminescence intensity vs photon energy obtained at 300 $\mathrm{K}$ under $45 \mathrm{~V}$ forward bias with 11-14 $\mathrm{mA}$ flowing through the diode.

ciencies measured at $150 \mathrm{~mA} / \mathrm{cm}^{2}$ are below $3 \times 10^{-5}$ photons per electron for all devices. Whereas the emission from the calcium/MEH-PPV/ITO LEDs (with efficiencies of approximately $1 \%$ photons per electron) is easily seen in a lighted room with a current flow of $2 \mathrm{~mA} / \mathrm{cm}^{2}$, LEDs made from P3OT require a dark background for easy viewing by eye. In our experience, the light emisssion is typically quite uniform over the entire device area. As the devices approach catastrophic failure, the current behavior becomes erratic, and the uniform emission gives out as bright spots take over. This breakdown is consistent with the microplasmas associated with avalanche multiplication or surface effects that enhance high-field breakdown. ${ }^{13}$

Figure 4 compares the efficiencies of devices formed with electrodes having different work functions. Increased quantum efficiency in MEH-PPV LEDs obtained with lower work function metals at the cathode suggests that poor minority carrier injection at the cathode accompanied

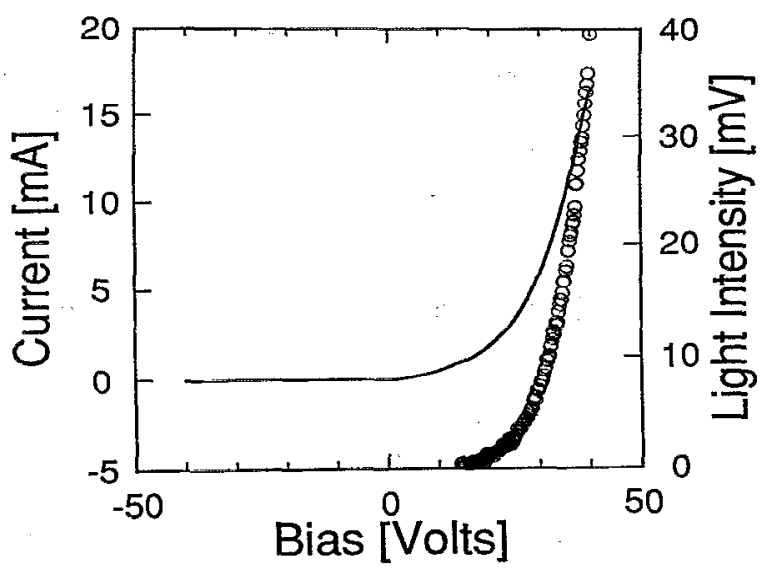

FIG. 2. Current flow (line) and luminescence intensity (circles) as a function of bias voltage for the polymer/calcium diode.

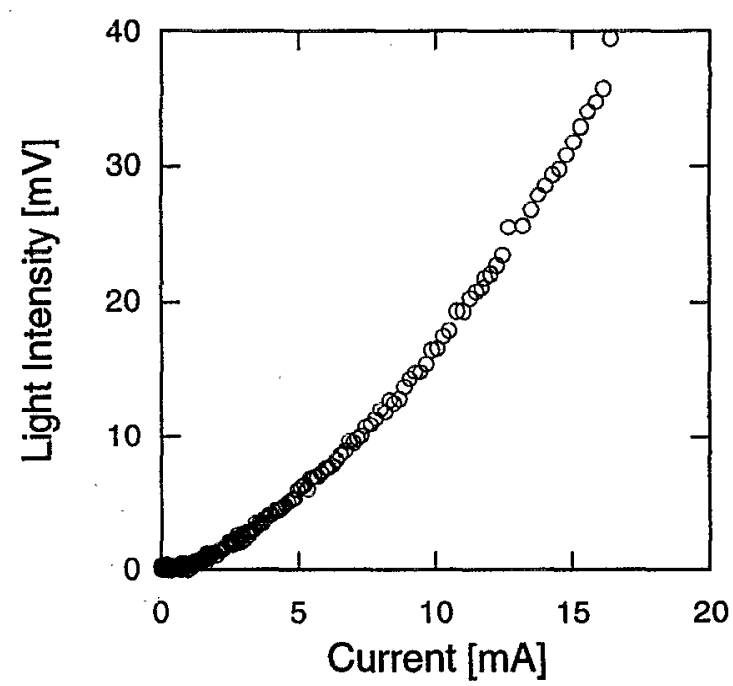

FIG. 3. Emitted light intensity recorded by calibrated Si photodiode vs current flowing through polymer LED with calcium electrode (circles).

by nonradiative relaxation of majority carriers limits device performance. ${ }^{14}$ Evidently, the condition is less dramatic for P3OT LEDs. Although calcium/P3OT/ITO LEDs display a lower turn-on voltage (field) than indium/ P3OT/ITO devices, we observe no improvement in efficiency. Note that the rectification ratio of the indium/ P3OT/ITO device ranges from 4500 to 3 , slightly lower values than those observed in the calcium/P3OT/ITO LED. For the indium/P3OT/ITO device, we observe a small amount of light emitted in the most extreme reverse bias.

The schematic energy band diagrams sketched in Fig. 5 are based on known values for the work functions of $\mathrm{Ca}$ $(2.87 \mathrm{eV})$ and In $(4.12 \mathrm{eV}) ;{ }^{15}$ we have assumed the electron affinity of ITO to be between the values known for $\mathrm{In}_{2} \mathrm{O}_{3}(4.34 \mathrm{eV})$ and $\mathrm{SnO}_{2}(4.75 \mathrm{eV}) .{ }^{16,17}$ Theoretical re-

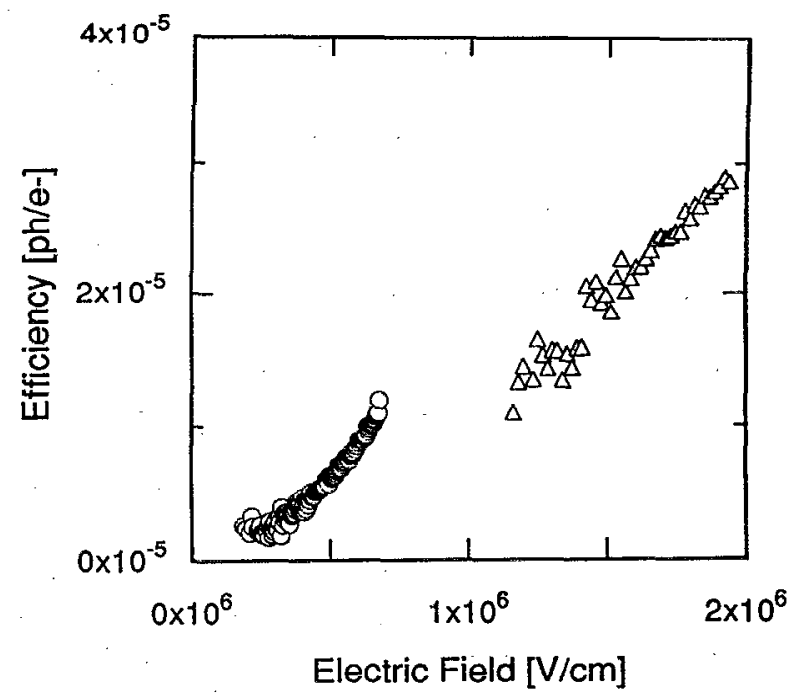

FIG. 4. External quantum efficiency vs electric field for LED with calcium electrode (circles) and indium electrode (triangles). 

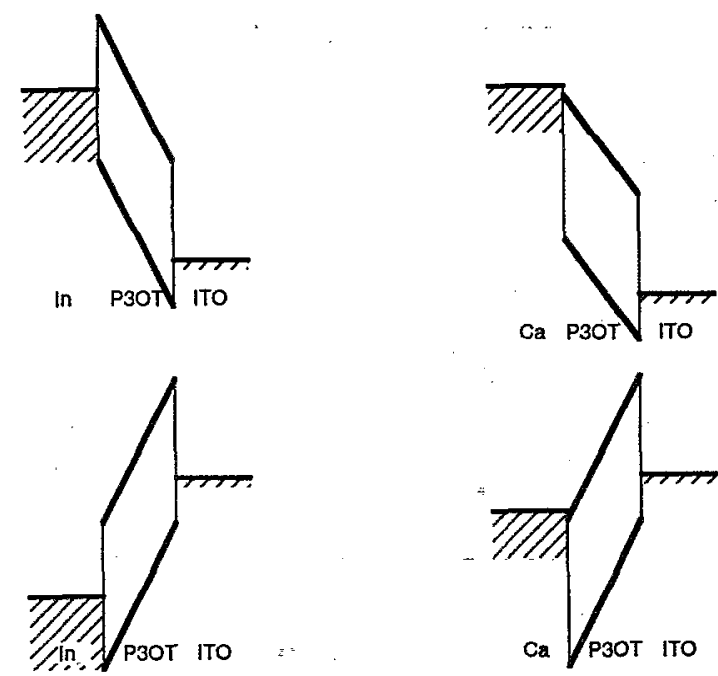

FIG. 5. Energy band diagrams for indium/P3OT/ITO and calcium/ P3OT/ITO LEDs under forward bias (above) and reversed bias (below).

sults estimate the ionization potential of polythiophene ${ }^{18}$ and poly (3-hexylthiophene) ${ }^{19}$ to be $5.0 \mathrm{eV}$, and the top of the valence band of poly (3-octylthiophene) should lie at almost the same energy. From the band gap of approximately $2 \mathrm{eV}$, we estimate the electron affinity at $3 \mathrm{eV}$. These crude band diagrams account for the observed rectification, including the slight improvement seen with the change in work function at the cathode. The diagrams suggest that hole injection could be improved by using an electrode with a work function larger than that of ITO.

For these P3OT diodes, it appears that nonradiative recombination limits the luminescence efficiency rather than carrier injection at the contacts. Because of the threefold multiplicity of the triplet exciton, at most $25 \%$ of the majority carriers can recombine radiatively via the singlet exciton state. ${ }^{4}$ It is also possible that bipolarons contribute to the current; the improbable radiative recombination of bipolaron carriers would act to diminish the luminescence efficiency. Most importantly, previous studies have demonstrated that trapped charges quench the photoluminescence in polythiophene and other polymers. ${ }^{20.21}$ In the P3OT material used for this study, extrinsic processes probably dominate the recombination; capacitance versus voltage measurements indicate nonuniform dopant concentrations in the range $10^{15}-10^{16} \mathrm{~cm}^{-3}$, higher by several orders of magnitude than impurity concentrations found in more efficient LEDs made from PPV. ${ }^{22}$ The P3OT oxidative coupling synthesis uses $\mathrm{FeCl}_{3}$, which is difficult to remove completely, even with subsequent purification. Since $\mathrm{FeCl}_{3}$ is also known to be a dopant for the polythiophenes, quite likely residual $\mathrm{FeCl}_{3}$ is present at levels sufficient to explain the capacitance measurements. This range provides only a lower limit on the number of possible recombination centers, because the capacitance does not measure neutral defects which can provide additional nonradiative recombination centers. Reducing extrinsic defects in P3OT should make possible more efficient electroluminescence.

The temperature dependence of indium/P3OT/ITO

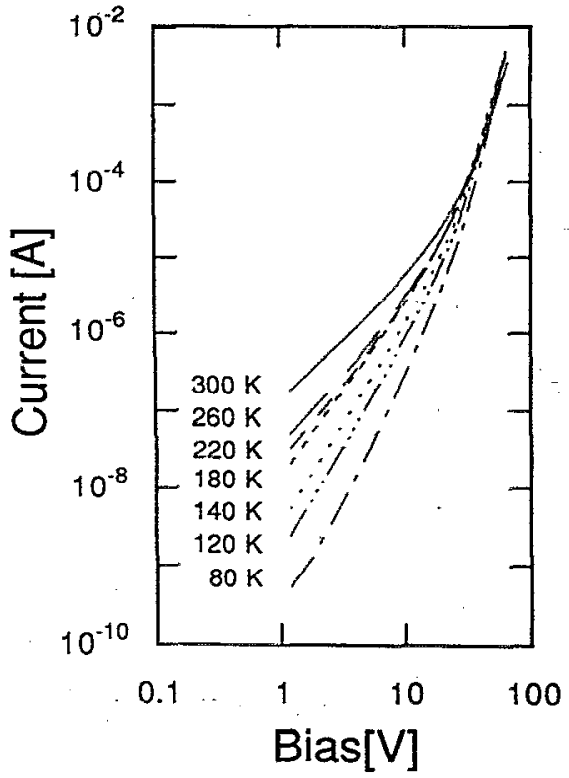

FIG. 6. Current vs bias voltage at temperatures between 80 and $300 \mathrm{~K}$ for indium/P3OT/ITO LED.

LED behavior provides information relevant to the charge injection and charge transport mechanisms. Figure 6 presents the current versus bias voltage data recorded during a temperature cycle from 300 to $80 \mathrm{~K}$. The bias was swept from 0 to $+66 \mathrm{~V}$ and then from 0 to $-66 \mathrm{~V}$ at each temperature. This strategy arose, after we observed that the devices fail catastrophically in reverse bias more often than in forward bias. Dividing the voltage sweep increases the likelihood of obtaining at least the forward bias data. Two conduction mechanisms stand out. In the range up to just above $30 \mathrm{~V}$, the current increases gradually with bias but increases approximately exponentially with temperature. In the highest voltage segment above $40 \mathrm{~V}$, the temperature dependence diminishes, and the current becomes nearly temperature independent.

During another temperature cycle, the bias was swept from -2 to $+2 \mathrm{~V}$ at each temperature; see Fig. $7(\mathrm{~A})$. In this low voltage range, two additional conduction mechanisms stand out. Parallel leakage current dominates below $100 \mathrm{pA}$, and, in the voltage range below $0.7 \mathrm{~V}$, an exponential segment is present above $140 \mathrm{~K}$. During other temperature cycles, the parallel leakage increases and restricts the extent of the exponential portion from below. Above $+1 \mathrm{~V}$, the onset of series resistance slows the exponential turn-on.

\section{MECHANISMS FOR CHARGE INJECTION AND TRANSPORT}

Numerous mechanisms previously identified in the study of organic and inorganic semiconductors can be considered to explain the origin of carrier injection and transport in polymer diodes. Thermally activated models include thermionic emission, thermionic field emission, diffusion theory, and combinations of the above. ${ }^{23,24}$ Tunneling can occur through a square barrier, ${ }^{25}$ triangular bar- 

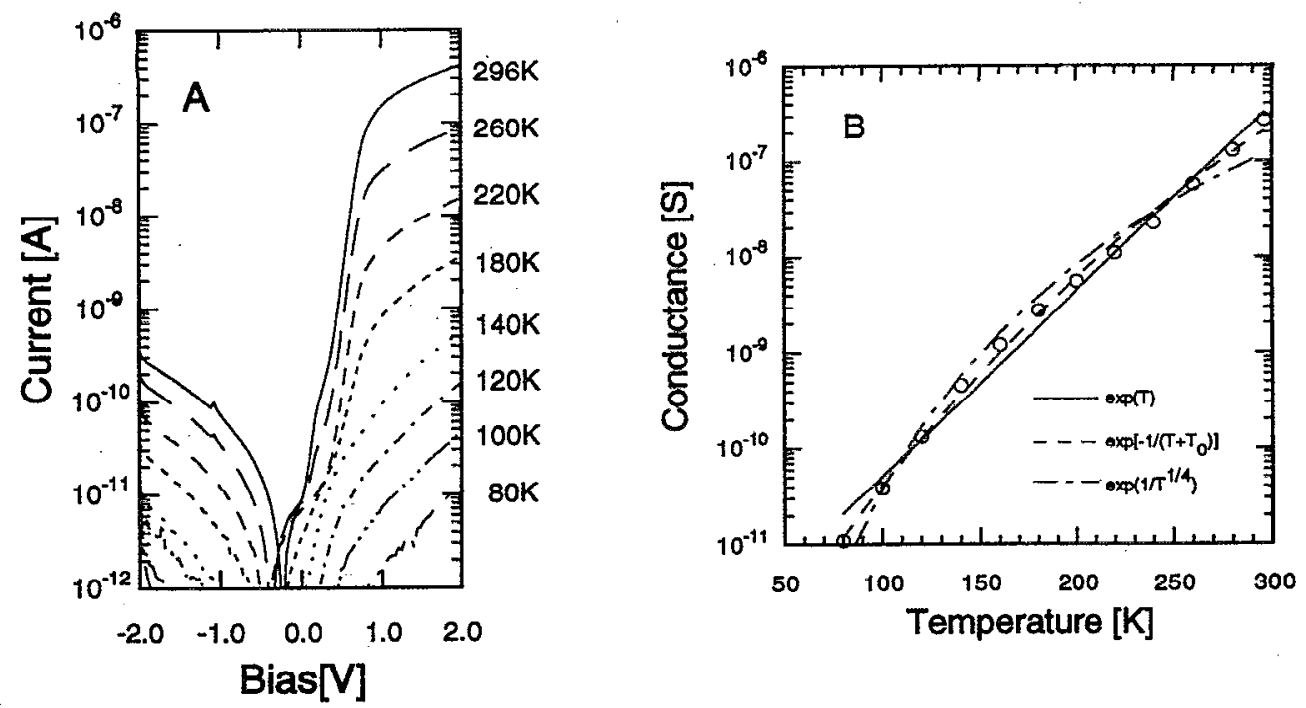

FIG. 7. Current vs bias voltage at temperatures between 80 and $300 \mathrm{~K}$ for indium/P3OT/ITO LED, panel A. Panel B shows conductivity between +1 and $+2 \mathrm{~V}$ bias obtained from data of panel A. Solid line fits to $\exp (T)$, dashed line fits $\exp \left[1 /\left(T+T_{0}\right)\right]$, and dot-dashed line fits $\exp \left(-1 / T^{1 / 4}\right)$.

rier, ${ }^{24,26}$ trapezoidal barrier, ${ }^{25}$ a barrier with nonuniform thickness, ${ }^{27-29}$ or across the band gap. ${ }^{30}$ Assisted by traps ${ }^{31}$ or thermal fluctuations, ${ }^{32-35}$ tunneling can have a more pronounced temperature dependence. With polymer devices, there also exists the possibility for avalanche multiplication, ${ }^{13,24}$ hopping conduction, ${ }^{36}$ ionic conduction, ${ }^{24}$ and space-charge-limited currents. ${ }^{37,38}$

The models with more parameters accommodate all of the data, but the broad flexibility of the models limits our ability to pin down the dominant mechanism unambiguously. In the lower ranges of voltage where the current has a strong temperature dependence, current increases exponentially with temperature. Figure $7(B)$ plots the conductivity obtained between +1 and $+2 \mathrm{~V}$ from the data in Fig. 7(A). The data fit best to the $\exp \left[-1 /\left(T+T_{0}\right)\right]$ dependence associated with thermal fluctuation induced tunneling through a parabolic barrier. ${ }^{32-35}$ Although the data can be fit quite accurately [see Fig. 7(B)], the model contains four independent parameters. The data fit better to an $\exp (T)$ behavior (seen in trap assisted tunneling) ${ }^{31}$ than to any expression of the form $\exp \left(-1 / T^{n}\right)$. The plot includes the best fit to $\exp \left(-1 / T^{n}\right)$ for $n=\frac{1}{4}$, bccause that expression (associated with three-dimensional variable range hopping) ${ }^{36}$ fits the data more closely than $n$ values relevant to the other models mentioned above. In the highest voltage range, the temperature dependence is minimal. Therefore, we dismiss models with an activated temperature dependence of the form $\exp \left(-1 / T^{n}\right)$, except in the low voltage range where the fit is still not perfect. Spacecharge-limited currents are ruled out since they would follow $V^{n}$, and they are unlikely for the range of carrier concentrations present.

This initial analysis eliminates all but the tunneling models. There are ample physical justifications to support each of the detailed tunneling models, but we have no independent evidence favoring one over the other; for example, we have no reason to prefer a square barrier to a triangular barrier or other barrier shapes. Multiparameter models such as the thermal fluctuation induced tunneling model are more flexible than P3OT. Most likely, the barrier is nonuniform over the area of the device (as a result of nonuniform thicknesses and interfacial layers) so that several forms of tunneling can contribute to the overall behavior.

Figure 8 shows the temperature dependence of the luminance versus current flow. In the temperature range measured, the luminescence steadily increases as more current flows. In addition, both brightness and efficiency improve at lower temperatures. The low temperature efficiency $(0.01 \%)$ is more than three times the room temperature value $(0.003 \%)$. Three ingredients contribute to the increased brightness at low temperature: charge injection, charge transport, and the radiative recombination efficiency of the polymer. The relative temperature inde-

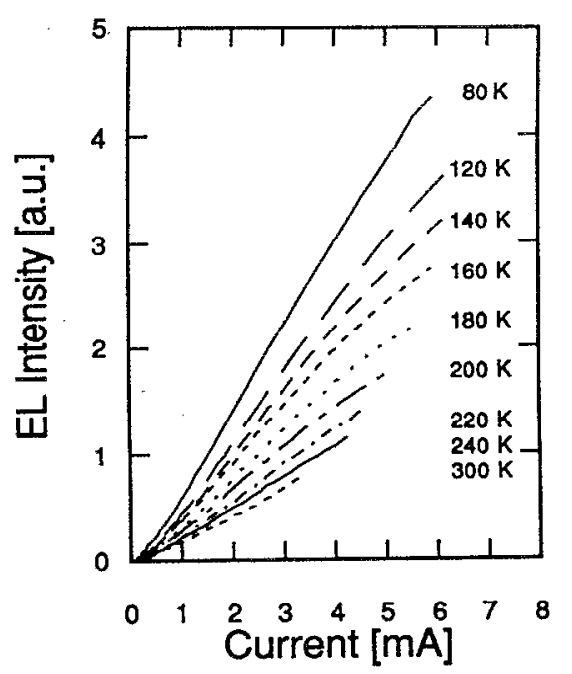

FIG. 8. Emitted light intensity recorded by calibrated Si photodiode vs current flowing through indium/P3OT/ITO LED at temperatures between 80 and $300 \mathrm{~K}$. 
pendence of the current versus voltage characteristics in the higher voltage range (where the brightest light is emitted) is consistent with tunneling dominating injection and transport. Figure 8 illustrates that slightly more current flows at low temperatures. It is certainly possible that the tunneling barrier height decreases at low temperature; for example, in the case of Zener tunneling, the barrier is the energy gap which does decrease slightly at low temperatures. Figure 8 also shows an improvement in quantum efficiency, indicating that radiative (or nonradiative) recombination becomes more (or less) efficient at low temperature. This conclusion is consistent with the temperature dependence of the photoluminescence and could result from a thermally activated nonradiative decay. ${ }^{39}$

\section{v. CONCLUSION}

We have studied the electroluminescence (EL) phenomenon, first observed with diodes fabricated from PPV or its derivatives, in a soluble conducting polymer with a different backbone structure, poly (3-octylthiophene). The temperature dependence of the current versus voltage characteristics of indium/P3OT/ITO diodes implies that charge injection takes place via tunneling. Contact potentials and Zener tunneling from band to band are possible origins of the tunneling barriers, and the likely formation of interfacial layers at the electrodes could create additional barriers. The results are similar to those obtained from diodes on poly (3-hexylthiophene). ${ }^{40}$ The intensity and efficiency of the electroluminescence increase at low temperatures, following the radiative recombination. The wide range of electroluminescence efficiency emphasizes the need for synthesis of ultrahigh purity semiconducting polymer materials.

\section{ACKNOWLEDGMENTS}

We thank Neste Corp. for providing the P3OT, UNIAX Corp. for loaning equipment used in device characterization, and $\mathrm{K}$. Voss for providing very helpful comments. The research was funded by the Office of Naval Research (N00014-83-K-0450).

'J. H. Burroughes, D. D. C. Bradley, A. R. Brown, R. N. Marks, K. Mackay, R. H. Friend, P. L. Burns, and A. B. Holmes, Nature 347, 539 (1990).

${ }^{2}$ D. Braun and A. J. Heeger, Appl. Phys. Lett. 58, 1982 (1991).

${ }^{3}$ P. L. Burn, A. B. Holmes, A. Kraft, D. D. C. Bradley, A. R. Brown, and R. H. Friend, J. Chem. Soc. Chem. Commun. 32 (1992).
${ }^{4}$ D. D. C. Bradley, A. R. Brown, P. L. Burn, R. H. Friend, A. B. Holmes, and A. Kraft, in Proceedings of the IWEPP, Kirchberg, 1991. ${ }^{5}$ T. Nakano, S. Doi, T. Noguchi, T. Ohnishi, and Y. Iyechika, European Patent Application \#91301416.3, 1991.

${ }^{6}$ P. L. Burn, A. Kraft, A. B. Holmes, A. R. Brown, D. D. C. Bradley, and R. H. Friend, Proceedings of the MRS Autumn Meeting, Boston, 1991.

${ }^{7}$ Y. Ohmori, M. Uchida, K. Muro, and K. Yoshino, Solid State Commun. 80, 605 (1991).

${ }^{8}$ G. Grem, Gr Leditzky, B. Ullrich, and G. Leising, Adv. Mater. 4, 36 (1992).

${ }^{9}$ T. W. Hagler, K. Pakbaz, K. Voss, and A. J. Heeger, Phys. Rev. B 44, 8652 (1991).

${ }^{10}$ B. C. Hess, J. Shinar, Q.-X. Ni, Z. Vardeny, and F. Wudl, Synth. Met. 28, C365 (1989).

${ }^{11}$ K. Yoshino, S. Nakajima, D. H. Park, and R.-i. Sugimoto, Jpn. J. Appl. Phys. 27, L716 (1988).

${ }^{12} \mathrm{~K}$. Yoshino, Synth. Met. 28, C669 (1989).

${ }^{13}$ C. D. Todd, Zener and Avalanche Diodes (Wiley, New York, 1970).

${ }^{14}$ D. Braun, A. J. Heeger, and H. Kroemer, J. Electron. Mater. 20, 945 (1991).

${ }^{15}$ D. R. Lide, Ed., CRC Handbook of Chemistry and Physics (CRC, Boca Raton, FL, 1991).

${ }^{16}$ G. Gustafsson, M. Sundberg, O. Inganas, and C. Svensson, J. Mol. Electron. 6, 105 (1990).

${ }^{17}$ D. L. Feucht, J. Vac. Sci. Technol. 14, 57 (1977).

${ }^{18} \mathrm{~J}$. L. Brédas, R. L. Elsenbaumer, R. R. Chance, and R. Silbey, J. Chrem. Phys. 78, 5656 (1983).

${ }^{19}$ B. Thémans, W. R. Salaneck, and J. L. Brédas, Synth. Met. 28, C359 (1989).

${ }^{20}$ D. D. C. Bradley and R. H. Friend, J. Phys: Condens. Matter 1, 3671 (1989).

${ }^{21}$ S. Hayashi, K. Kaneto, and K. Yoshino, Solid State Commun. 61, 249 (1987).

22 D. D. C. Bradley, seminar presented at the University of California, Santa Barbara, Aug. 16, 1991.

${ }^{23}$ E. H. Rhoderick and R. H. Williams, Metal-Semiconductor Contacts (Clarendon, Oxford, 1988).

${ }^{24}$ S. M. Sze, Physics of Semiconductor Devices (Wiley, New York, 1981).

${ }^{25}$ J. G. Simmons, J. Appl. Phys. 34, 2581 (1963).

${ }^{26}$ R. H. Fowler and L. Nordheim, Proc. Roy. Soc. London 119, 173 (1928).

${ }^{27}$ Z. Hurych, Solid-State Electron. 9, 967 (1966)...

${ }^{28} \mathrm{Z}$. Hurych, Solid-State Electron: 13, 683 (1970).

${ }^{29}$ C. K. Chow, J. Appl. Phys. 34, 2599 (1963).

${ }^{30}$ A. R. Riben and D. L. Feucht, Solid-State Electron. 9, 1055 (1966).

${ }^{31}$ A. R. Riben and D. L. Feucht, Int. J. Electron. 20, 583 (1966).

${ }^{32}$ P. Sheng, Phys. Rev. B 21, 218 (1980).

${ }^{33}$ K. Väkiparta, J. Moulton, A. J. Heeger, P. Smith, H. Isotalo, H. Stubb, and M. Loponen, Synth. Met. 41-43, 903 (1991):

${ }^{34}$ J. Voit and H. Büttner, Solid State Commun. 67, 1233 (1988).

${ }^{35}$ T. Schimmel, G. Denninger, W. Riess, J. Voit, M. Schwoerer, W. Schoepe, and H. Naarmann, Synth. Met. 28, D11 (1989).

${ }^{36}$ N. F. Mott and E. A. Davis, Electronic Processes In Non-Crystalline Materials (Clarendon, Oxford, 1979).

${ }^{37}$ M. A. Lampert and P. Mark, Current Injection In Solids (Academic, New York, 1970).

${ }^{38}$ P. Mark and W. Helfrich, J. Appl. Phys. 33, 205 (1962).

${ }^{39}$ J. Rühe, N. F. Colaneri, D. D. C. Bradley, R. H. Friend, and G. Wegner, J. Phys.: Condens. Matter 2, 5465 (1990).

${ }^{40}$ H. Tomozawa, D. Braun, S. D. Phillips, R. Worland, A. J. Hecger, and H. Kroemer, Synth. Met. 28, C687 (1989). 